\title{
ARTICLE
}

Received 4 Sep 2015 | Accepted 20 Jan 2016 | Published 24 Feb 2016

DOI: $10.1038 /$ ncomms10801

OPEN

\section{Creating single-atom Pt-ceria catalysts by surface step decoration}

Filip Dvořák ${ }^{1, \star}$, Matteo Farnesi Camellone ${ }^{2, \star}$, Andrii Tovt ${ }^{1}$, Nguyen-Dung Tran ${ }^{2,3}$, Fabio R. Negreiros ${ }^{2, \dagger}$, Mykhailo Vorokhta1, Tomáš Skála, Iva Matolínová', Josef Mysliveček', Vladimír Matolín ${ }^{1}$ \& Stefano Fabris²,3

Single-atom catalysts maximize the utilization of supported precious metals by exposing every single metal atom to reactants. To avoid sintering and deactivation at realistic reaction conditions, single metal atoms are stabilized by specific adsorption sites on catalyst substrates. Here we show by combining photoelectron spectroscopy, scanning tunnelling microscopy and density functional theory calculations that Pt single atoms on ceria are stabilized by the most ubiquitous defects on solid surfaces-monoatomic step edges. $\mathrm{Pt}$ segregation at steps leads to stable dispersions of single $\mathrm{Pt}^{2+}$ ions in planar $\mathrm{PtO}_{4}$ moieties incorporating excess $\mathrm{O}$ atoms and contributing to oxygen storage capacity of ceria. We experimentally control the step density on our samples, to maximize the coverage of monodispersed $\mathrm{Pt}^{2+}$ and demonstrate that step engineering and step decoration represent effective strategies for understanding and design of new single-atom catalysts.

\footnotetext{
${ }^{1}$ Charles University in Prague, Faculty of Mathematics and Physics, V Holešovičkách 2, Prague 18000, Czech Republic. ${ }^{2}$ CNR-IOM DEMOCRITOS, Istituto Officina dei Materiali, Consiglio Nazionale delle Ricerche, Via Bonomea 265, Trieste 34136, Italy. ${ }^{3}$ SISSA, Scuola Internazionale Superiore di Studi Avanzati, Via Bonomea 265, Trieste 34136, Italy. ${ }^{\star}$ These authors contributed equally to this work. †Present address: Universidade Federal do ABC. Av. dos Estados, 5001 Bairro Bangu, Santo André SP CEP 09210-580, Brasil. Correspondence and requests for materials should be addressed to J.M. (email: josef.myslivecek@mff.cuni.cz) or to S.F. (email: fabris@democritos.it).
} 
S ingle-atom catalysts represent the limiting realization of supported metal catalysts with metal load ultimately dispersed as single atoms ${ }^{1,2}$. This maximizes the utilization of supported metals and helps development of sustainable catalytic technologies for renewable energies and environmental applications with reduced precious metal contents ${ }^{3,4}$. A central prerequisite for understanding and knowledge-based design of single-atom catalysts is the identification of specific adsorption sites on catalyst supports that provide the stabilization of single metal atoms under reaction conditions at elevated temperatures and pressures. For oxide supports, understanding specific adsorption sites presently concentrates on low-index oxide facets ${ }^{5-9}$. Single-atom catalysts are, however, nanostructured large-area materials; thus, a question arises whether single supported atoms can be stabilized at defect sites of nanostructured oxide supports.

Highly dispersed platinum (Pt) ions on ceria qualify as single-atom catalysts ${ }^{2}$ and hold a promise of radical reduction of Pt load in critical large-scale catalytic applications-hydrogen production $^{3}$, three-way catalytic converters ${ }^{10}$ and fuel cells ${ }^{11}$. Ceria surfaces provide a limited amount of low coordinated surface sites where $\mathrm{Pt}^{2+}$ ions can adsorb and remain stable in real applications $2,3,10,11$. Recent studies on large-area ceria samples identify the necessity of nanostructuring the ceria substrates for obtaining supported $\mathrm{Pt}^{2+}$ ions $s^{2,4}$ and propose a square-planar $\mathrm{PtO}_{4}$ unit as a $\mathrm{Pt}^{2+}$-containing surface moiety ${ }^{4}$. In the present model study on the single crystalline $\mathrm{CeO}_{2}(111)$ surface, we demonstrate that single-ion dispersions of $\mathrm{Pt}^{2+}$ are stabilized at monolayer (ML)-high ceria step edges. $\mathrm{Pt}^{2+}$ ions at step edges are located in $\mathrm{PtO}_{4}$ units that can be considered the elementary building blocks of $\mathrm{Pt}^{2+} /$ ceria single-atom catalysts. The $\mathrm{PtO}_{4}$ units incorporate excess $\mathrm{O}$ and can act as oxygen source for redox reactions. Besides clarifying the nature of $\mathrm{Pt}^{2+}$ stabilization on ceria, our study demonstrates the importance of step edges-the most common surface defects on oxide supports ${ }^{12}$-for single-atom catalyst stabilization. We experimentally adjust the step density on the ceria supports for maximizing the load of monodispersed $\mathrm{Pt}^{2}+$ ions. This identifies step engineering ${ }^{13}$ and step decoration ${ }^{14,15}$ as advanced techniques for designing new single-atom catalysts.

\section{Results}

Pt deposits on highly defined $\mathrm{CeO}_{2}(111)$ surfaces. The experiments were performed on model $\mathrm{CeO}_{2}(111)$ surfaces prepared as 20 to $40 \AA$ thick ceria films on $\mathrm{Cu}(111)$ using procedures that allow adjusting the density of ML-high steps ${ }^{16}$ and the density of surface oxygen vacancies on ceria surface ${ }^{17}$. On these highly defined surfaces, we deposit $0.06 \mathrm{ML}$ of $\mathrm{Pt}$, anneal at $700 \mathrm{~K}$ in ultra-high vacuum (UHV) and observe stabilization of $\mathrm{Pt}^{2+}$ species and/or nucleation of Pt clusters with scanning tunnelling microscopy (STM) and with photoelectron spectroscopy (PES). Deposition and annealing of $\mathrm{Pt}$ on $\mathrm{CeO}_{2}(111)$ surfaces containing low concentrations of defectsML-high steps and surface oxygen vacancies (Fig. 1a)-yield
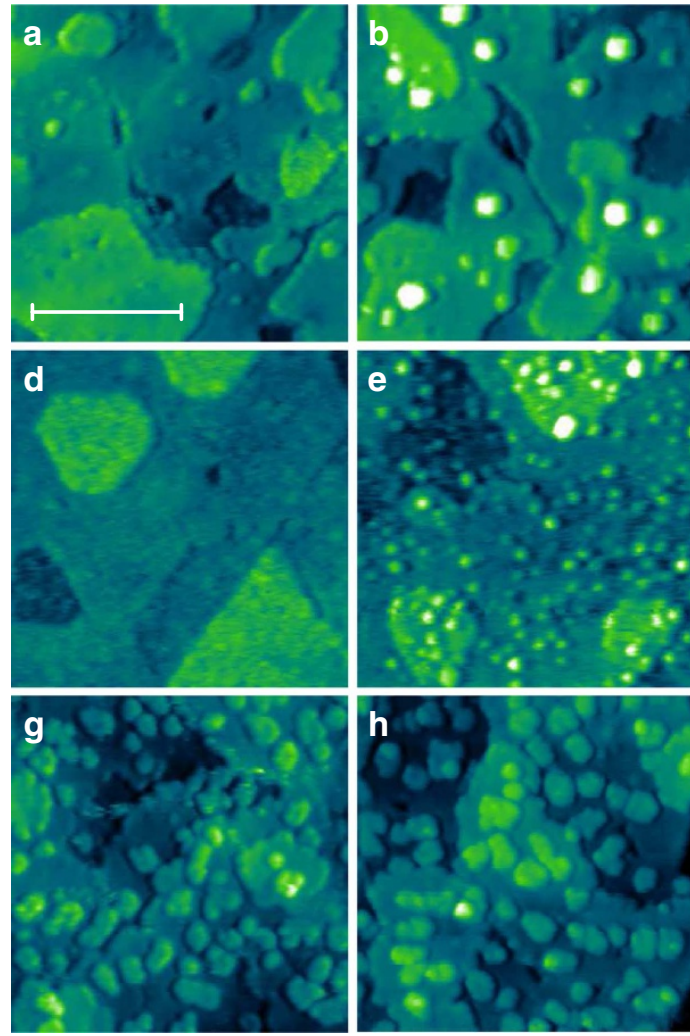

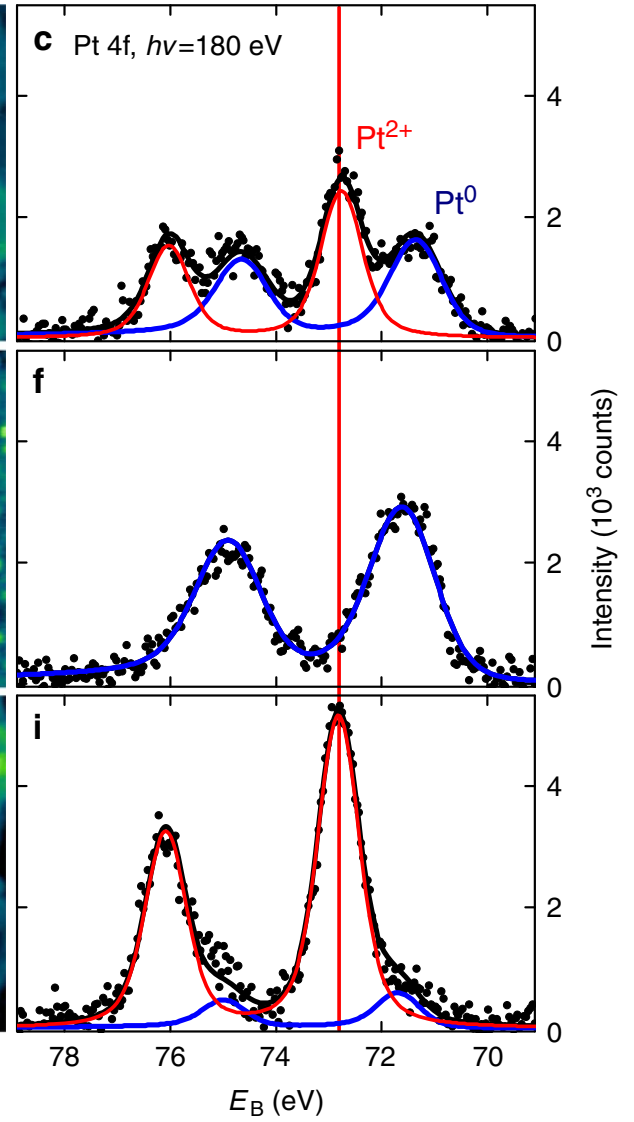

Figure 1 | Nucleation of $\mathbf{P t}$ and stabilization of $\mathbf{P t}^{\mathbf{2}+}$ on ceria surfaces containing controlled amount of surface defects. (a-c) $\mathrm{CeO} \mathrm{O}_{2}(111)$ surface with low density of surface oxygen vacancies and $\mathrm{ML}$-high steps. (d-f) $\mathrm{CeO}_{1.7}$ surface with increased density of surface oxygen vacancies. (g-i) $\mathrm{CeO}_{2}(111)$ surface with increased density of ML-high steps. (a,d,g) STM images of clean surfaces before deposition of Pt. (b,e, h) STM images after deposition of $0.06 \mathrm{ML}$ Pt and annealing at $700 \mathrm{~K}$ in UHV. All STM images $45 \times 45 \mathrm{~nm}^{2}$, tunnelling current $25-75 \mathrm{pA}$, sample bias voltage $2.5-3.5 \mathrm{~V}$. Scale bar, $20 \mathrm{~nm}$ (a). $(\mathbf{c}, \mathbf{f}, \mathbf{i})$ PES spectra of the Pt deposit after annealing. All PES spectra were acquired with photon energy $h v=180 \mathrm{eV}$ (black points). Fits indicate metallic $\left(\mathrm{Pt}^{0}\right.$, blue line) and ionic $\left(\mathrm{Pt}^{2+}\right.$, red line) contributions to $\mathrm{Pt} 4 \mathrm{f}$ signal. $E_{\mathrm{B}}$ is the photoelectron binding energy. 
metallic $\mathrm{Pt}^{0}$ clusters (Fig. 1b,c) coexisting with ionic $\mathrm{Pt}^{2+}$ species (Fig. 1c). To determine whether the charge of the supported $\mathrm{Pt}$ species is selectively induced by a specific defect type, we repeat the experiment varying independently the amount of surface $\mathrm{O}$ vacancies-up to $0.16 \mathrm{ML}$, creating $\mathrm{CeO}_{1.7}$ surface (Fig. 1d-f) -and the amount of ML-high steps on the $\mathrm{CeO}_{2}(111)$ surface- up to $0.15 \mathrm{ML}$ (Fig. 1g-i). We observe that surface oxygen vacancies do not promote the dispersion of $\mathrm{Pt}^{2+}$ species but lead to small metallic $\mathrm{Pt}^{0}$ clusters (Fig. $\left.1 \mathrm{e}, \mathrm{f}\right)^{18}$. On the other hand, the increased step density leads to almost complete oxidation of the Pt deposit to $\mathrm{Pt}^{2+}$ (Fig. 1h,i) proving that step edges selectively promote the stabilization of $\mathrm{Pt}^{2+}$ species. Detailed STM images allow to exclude formation of three-dimensional and twodimensional $\mathrm{PtO}_{x}$ clusters (Supplementary Fig. 1), and allow to conclude that $\mathrm{Pt}^{2+}$ species are incorporated in the ceria step edges. Nucleation of $\mathrm{Pt}^{0}$ clusters and stabilization of $\mathrm{Pt}^{2+}$ species represent concurrent processes. Differently to $\mathrm{Pt}^{0}$ clusters (Fig. 1b,e), $\mathrm{Pt}^{2+}$ species at the step edges are not discernible in empty states STM imaging ( $c f$. Fig. 1g,h without and with Pt deposit) because of their electronic structure. STM imaging in occupied states on metal-supported ceria is unavailable ${ }^{19}$.

The possibility to adjust the density of ML-high steps on the model $\mathrm{CeO}_{2}(111)$ surfaces ${ }^{16}$ allows us to obtain a quantitative correlation between the step density and the amount of $\mathrm{Pt}^{2+}$ species. We prepare $\mathrm{CeO}_{2}(111)$ samples with step density between 0.06 and $0.20 \mathrm{ML}^{16}$ and deposit 0.06 or $0.18 \mathrm{ML} \mathrm{Pt}$ at $300 \mathrm{~K}$. Parameters of the prepared samples are summarized in Supplementary Table 1. After annealing at $700 \mathrm{~K}$ the amount of Pt stabilized in the form of $\mathrm{Pt}^{2+}$ is determined by PES. For quantification, all relevant parameters-the density of ceria steps, deposited amount of $\mathrm{Pt}$ and amount of stabilized $\mathrm{Pt}^{2+}$-are expressed in ML where $1 \mathrm{ML}$ corresponds to the density of Ce atoms on the $\mathrm{CeO}_{2}(111)$ surface, that is, $7.9 \times 10^{14} \mathrm{~cm}^{-2}$. The density of steps is defined as the density of Ce atoms located at the ceria step edges ${ }^{20}$.

The amount of $\mathrm{Pt}^{2+}$ ions as a function of the step density is plotted in Fig. 2a. For the higher Pt coverage 0.18 ML, the analysis reveals a linear dependence between the amount of stabilized $\mathrm{Pt}^{2+}$ ions and the step density (Fig. 2a, blue symbols), confirming the activation of $\mathrm{Pt}$ oxidation to $\mathrm{Pt}^{2+}$ and the localization of $\mathrm{Pt}^{2}+$ at the surface steps. The highest step density $0.20 \mathrm{ML}$ allows converting up to $80 \%$ of the $\mathrm{Pt}$ deposit to $\mathrm{Pt}^{2+}$. The degree of oxidation of the Pt deposit increases with decreasing the amount of deposited Pt to $0.06 \mathrm{ML}$ (Fig. 2a, black symbols). In this case, up to $90 \%$ of $\mathrm{Pt}$ converts to $\mathrm{Pt}^{2+}$. The concentrations of ceria step edges and the amount of $\mathrm{Pt}^{2+}$ stabilized on the surface obey a classical supply-and-demand scenario characteristic for single-atom catalysts ${ }^{1,4,10}$ : when sufficient step edges, the amount of oxidized $\mathrm{Pt}^{2+}$ is limited by the amount of deposited Pt. Otherwise, the amount of oxidized $\mathrm{Pt}^{2+}$ is limited by the step density regardless of the amount of deposited Pt. The Pt deposit exceeding the available step sites cannot be oxidized and nucleates as metallic $\mathrm{Pt}^{0}$ clusters on the surface. Besides the high oxidative power of the step edges towards $\mathrm{Pt}$, our quantitative analysis reveals also the capacity of ceria step edges to accommodate a high density of $\mathrm{Pt}^{2+}$ ions. Up to $0.16 \mathrm{ML}$ of $\mathrm{Pt}^{2+}$ ions can be stabilized by the sample containing 0.18 ML of Pt deposit and $0.20 \mathrm{ML}$ of steps (Fig. 2a, blue symbols). This corresponds to $80 \%$ of the step-edge sites being occupied by $\mathrm{Pt}^{2+}$. In the whole range of the step densities between 0.06 and $0.20 \mathrm{ML}$, the occupation of the step-edge sites by $\mathrm{Pt}^{2+}$ varies between 50 and $80 \%$.

Stability and charge state of $\mathrm{Pt}^{2+} / \mathrm{CeO}_{2}(111)$ samples. The necessity of annealing the Pt deposit on ceria in UHV at $700 \mathrm{~K}$ for obtaining $\mathrm{Pt}^{2+}$ stabilization in our experiment indicates the

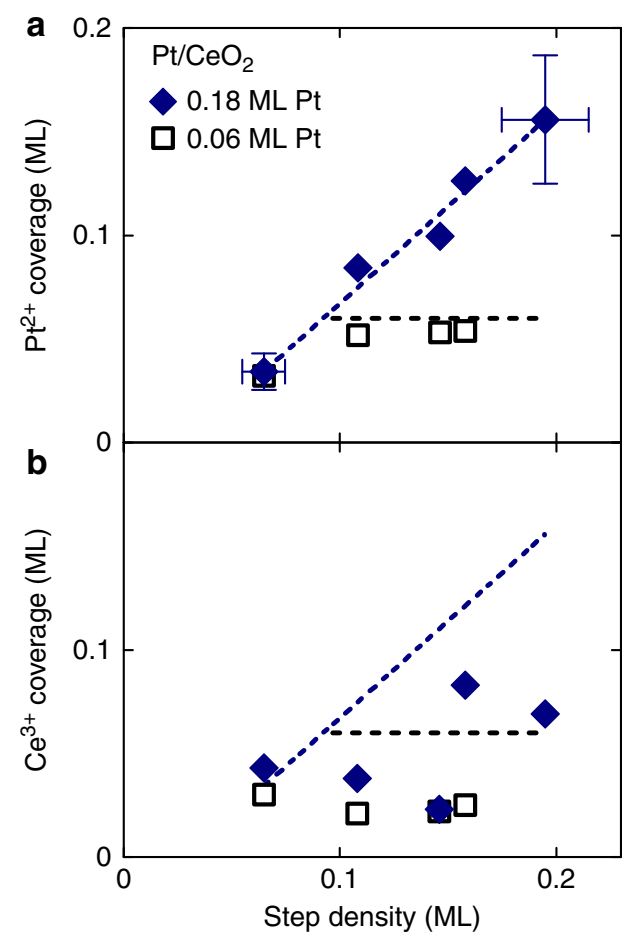

Figure 2 | Capacity of stepped $\mathrm{CeO}_{2}(111)$ surface to accommodate $\mathrm{Pt}^{2}+$. (a) Amount of $\mathrm{Pt}^{2}+$ stabilized on $\mathrm{CeO}_{2}$ (111) substrates with different density of steps for $0.18 \mathrm{ML}$ (blue symbols) and $0.06 \mathrm{ML}$ (black symbols) of deposited platinum. Pt not stabilized in the form of $\mathrm{Pt}^{2+}$ remains metallic. Lines represent guides to the eyes. Blue line is a linear fit of $0.18 \mathrm{ML}$ Pt data. Black line represents the maximum achievable amount of $\mathrm{Pt}^{2}+$ in the case of $100 \%$ oxidation of Pt for $0.06 \mathrm{ML}$ of deposited Pt. (b) Reduction of the ceria surface accompanying the stabilization of $\mathrm{Pt}^{2}+$ ions determined by resonant PES expressed as a coverage of the surface by $\mathrm{Ce}^{3+}$ ions. Lines represent guides to the eyes from a and indicate the $\mathrm{Pt}^{2+}$ concentration. The $\mathrm{Ce}^{3+}$ concentration is lower or equal to the $\mathrm{Pt}^{2+}$ concentration on all samples.

activated nature of Pt segregation at the ceria steps and oxidation, and implies considerable thermal stability of $\mathrm{Pt}^{2+}$ ions on ceria. High-temperature annealing represents a prerequisite for obtaining $\mathrm{Pt}^{2+}$ ions also in the experiments on large-area nanostructured ceria samples ${ }^{3,4}$. Once created, $\mathrm{Pt}^{2+}$ ions remain stable on repeated annealing at $700 \mathrm{~K}$ in UHV. The $\mathrm{Pt}^{2+}$ ions in our experiment also remain stable on adsorption and thermal desorption of CO in UHV (Supplementary Fig. 2), or on exposure to air at ambient conditions (Supplementary Fig. 3).

Parallel to the charge state of the Pt deposit we determine the charge state of the $\mathrm{CeO}_{2}$ support, in particular the concentration of surface $\mathrm{Ce}^{3+}$ ions that is indicative of reduction of the ceria surface. Contrary to the case of stabilizing $\mathrm{Ni}^{2+}$ ions on ceria ${ }^{21}$, we observe that $\mathrm{Pt}$ oxidation during annealing is not accompanied by a corresponding reduction of $\mathrm{CeO}_{2}(111)$ surface (Fig. 2b). This rules out the direct participation of ceria into the observed $\mathrm{Pt}$ oxidation at steps and indicates the involvement of other oxidizing agents in the $\mathrm{Pt}^{2+}$ stabilization, such as excess oxygen atoms. In the UHV environment of our experiments, the eligible source of excess oxygen can be water adsorbing in sub-ML amounts from background atmosphere (Supplementary Fig. 4) and undergoing dissociation on reduced ceria and $\mathrm{Pt} /$ ceria substrates ${ }^{22,23}$. In the large-area $\mathrm{Pt}^{2+} / \mathrm{CeO}_{2}$ catalysts displaying high concentration of $\mathrm{Pt}^{2+}$ ions and exceptional redox reactivity, excess $\mathrm{O}$ atoms may be incorporated during the synthesis that proceeds in air ${ }^{3,10}$. 
Segregation of $\mathrm{Pt}$ at $\mathrm{CeO}_{2}(111)$ step edges. Ab initio density functional theory (DFT) calculations allow to interpret the above experimental results. We calculate the segregation thermodynamics and the atomic and electronic structures of $\mathrm{Pt}$ atoms in representative adsorption sites on $\mathrm{CeO}_{2}(111)$ surfaces. The results
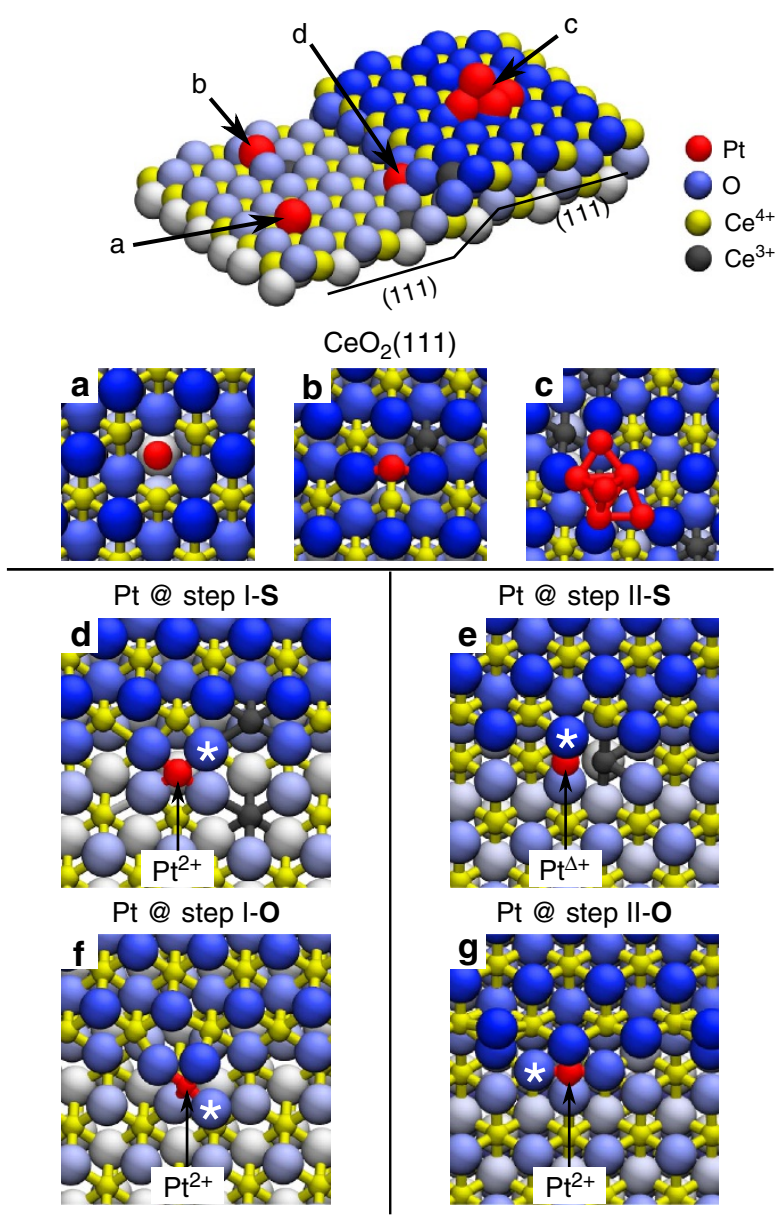

Figure 3 | Pt adsorption sites on the $\mathrm{CeO}_{2}$ (111) surface obtained from DFT calculations. (a) Pt adatom in a surface $\mathrm{O}$ vacancy, (b) on the stoichiometric $\mathrm{CeO}_{2}(111)$ terrace and (c) supported $\mathrm{Pt}_{6}$ cluster. (d) Pt adatom at the stoichiometric step I (step I-S) and (e) at the stoichiometric step II (step II-S). (f) Pt adatom at the step I with excess O (step I-O) and $(\mathbf{g})$ at the step II with excess $\mathrm{O}$ (step II-O). Binding energies and Bader charges are summarized in Table 1. $(\mathbf{d}-\mathbf{g})$ The * symbol denotes the $O$ atom removed to calculate the $\mathrm{O}$ vacancy formation energy reported in Table 2. for the lowest-energy configurations are summarized in Fig. 3 and Table 1. The model adsorption sites include oxygen vacancies (Fig. 3a), regular sites (Fig. 3b) and Pt clusters ${ }^{24}$ (Fig. 3c) on the $\mathrm{CeO}_{2}(111)$ terrace, as well as two low-energy ML-high steps, which we label following ref. 25 as step I (Fig. 3d) and step II (Fig. 3e). A detailed list of the systems considered in the DFT analysis is reported in the Supplementary Note 1 . The steps I and II represent the preferred types of steps at the $\mathrm{CeO}_{2}(111)$ surfaces at temperatures $<1,000 \mathrm{~K}$ (ref. 25). On our experimental samples, the steps I and II appear in equal proportion as evidenced from the absence of triangularly shaped islands in Fig. $1 \mathrm{a}, \mathrm{g}^{25,26}$.

In agreement with the experiment, our calculations predict the preferential segregation of $\mathrm{Pt}$ adatoms at the steps I and II, independently on the local step geometry and stoichiometry. The binding energies of $\mathrm{Pt}$ at the steps are 1.6-3.4 eV higher than at stoichiometric or defective (111) terraces (Table 1). This driving force for $\mathrm{Pt}$ segregation at the steps is in qualitative agreement with recent calorimetric studies of other metal clusters on $\mathrm{CeO}_{2}(111)^{27,28}$, see the Supplementary Discussion. The particular binding energy and the charge state of $\mathrm{Pt}$ atoms at the steps depend on both the local step geometry and stoichiometry (Table 1). In the following, we show that a good agreement with all the experimental observations can only be achieved when considering segregation of $\mathrm{Pt}$ at steps in the presence of excess $\mathrm{O}$ (calculations denoted as $\mathrm{O}$ ), while $\mathrm{Pt}$ segregation at stoichiometric steps (denoted S) exhibits significant discrepancies.

Pt at stoichiometric $\mathrm{CeO}_{2}(111)$ step edges. Pt segregation on step I-S yields $\mathrm{Pt}^{2}+$ species that are coordinated by four lattice $\mathrm{O}$ atoms in a characteristic $\mathrm{PtO}_{4}$ planar unit (Fig. 3d). The $\mathrm{PtO}_{4}$ unit is remarkably similar to that one proposed for Pt-doped ceria nanoparticles 4 and for surface reconstructions of $\mathrm{Pd}$-ceria systems ${ }^{29}$. Instead, the different atomic structure of the step II$\mathrm{S}$ edge prevents the formation of $\mathrm{PtO}_{4}$ units, hinders the full $\mathrm{Pt}$ oxidation to $\mathrm{Pt}^{2+}$ and yields weakly oxidized $\mathrm{Pt}^{\Delta+}$ species (Fig. 3e). Calculation results presented in Fig. 3d-g correspond to the $\mathrm{Pt}$ coverage at the steps $1 / 3$ (1 $\mathrm{Pt}$ atom per $3 \mathrm{Ce}$ step-edge atoms). For interpreting the capacity of the ceria step edges to accommodate a high density of $\mathrm{Pt}^{2+}$ ions, we calculate the adsorption of $\mathrm{Pt}$ at the ceria steps with increasing Pt coverage at the steps (Fig. 4), ranging from $1 / 3$ to 1 (1 Pt atom per $1 \mathrm{Ce}$ stepedge atom). On the step I-S, the maximum coverage of $\mathrm{Pt}^{2+}$ species is $2 / 3$ (Fig. 4a). Higher $\mathrm{Pt}^{2+}$ coverages are unattainable and lead to nucleation of metallic Pt clusters, due to the large strain buildup resulting from long sequences of interconnected $\mathrm{PtO}_{4}$ step units (Fig. 4b). On the step II-S, metallic $\mathrm{Pt}^{0}$ species appear already for a coverage higher than $1 / 3$ (Fig. 4c). Thus, on

Table 1 | Properties of $\mathrm{Pt}$ on $\mathrm{CeO}_{2}$ (111) obtained from DFT calculations.

\section{Pt adsorption site}

Pt @ O vacancy

Pt @ $\mathrm{CeO}_{2}(111)$

$\mathrm{Pt}_{6} @ \mathrm{CeO}_{2}(111)$

Pt @ step II-S

Pt @ step 1-O

Pt @ step II-O

Pt bulk
Pt@step I-S

\section{Binding energy (eV)}

2.8

3.3

4.4

5.0

5.1

6.6

6.7

$5.5^{*}$
Formal charge

$\begin{array}{cc}\mathrm{Pt}^{\Delta-} & 10.9 \\ \mathrm{Pt}^{\Delta+} & 9.7 \\ \mathrm{Pt}^{\mathrm{O}} & 9.9 \\ \mathrm{Pt}^{2+} & 9.2 \\ \mathrm{Pt}^{\Delta+} & 9.7 \\ \mathrm{Pt}^{2+} & 8.6 \\ \mathrm{Pt}^{2+} & 9.0 \\ \mathrm{Pt}^{0} & 10.0\end{array}$

DFT, density functional theory; Pt, platinum; I-S and II-S, stoichiometric step types I and II; I-O and II-O, step types I and II with excess O atoms.

Results for the binding energies per atom, formal charges and Bader charges of $\mathrm{Pt}$ adatoms at the adsorption sites displayed in Fig. 3a-g. The binding energy for the $\mathrm{Pt}{ }_{6}$ cluster is the total binding energy divided by the number of $\mathrm{Pt}$ atoms. For reference, $\mathrm{Pt}$ bulk values are given in the last line.

*Bulk cohesive energy. 


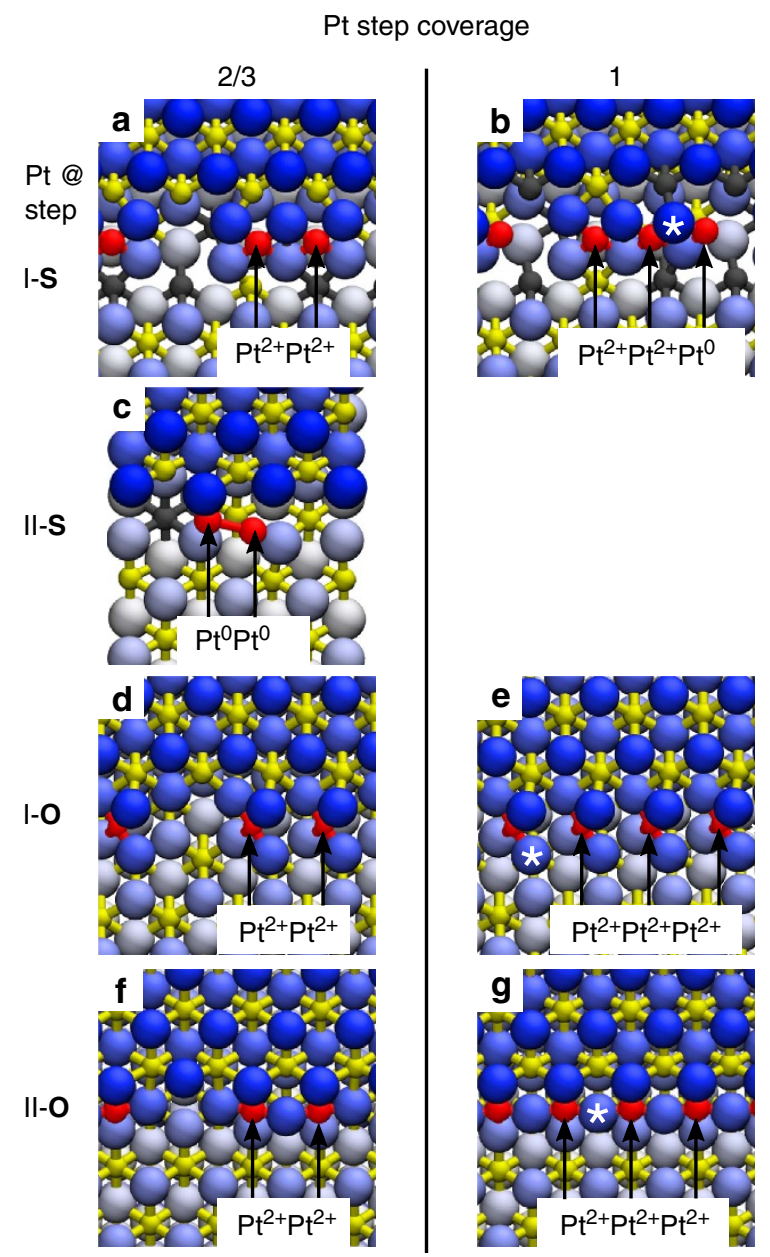

Figure 4 | Capacity of the $\mathrm{CeO}_{2}(111)$ step edges to accommodate $\mathbf{P t}^{2+}$ ions obtained from DFT calculations. Calculated top views of the Pt binding to the steps I-S $(\mathbf{a}, \mathbf{b})$, step II-S (c), step I-O (d,e $)$ and the step II-O $(\mathbf{f}, \mathbf{g})$ for Pt step coverage $2 / 3(\mathbf{a}, \mathbf{c}, \mathbf{d}, \mathbf{f})$ and $1(\mathbf{b}, \mathbf{e}, \mathbf{g})$. At the step I-S, the limiting coverage of $\mathrm{Pt}^{2+}$ is $2 / 3(\mathbf{a})$, additional $\mathrm{Pt}$ attaches to step edge as $\mathrm{Pt}^{0}$ (b). At the step II-S, the $\mathrm{Pt}^{2+}$ coverage is 0 . Pt atoms attach as weakly ionized $\mathrm{Pt}^{\Delta+}$ and readily form metallic dimers (c) and clusters. On both steps I-O and II-O, excess oxygen can stabilize ionic $\mathrm{Pt}^{2}+$ at step edges as single ions appearing isolated or in groups up to $100 \%$ step coverage $(\mathbf{d}-\mathbf{g})$. The * symbol denotes the $\mathrm{O}$ atom removed to calculate the $\mathrm{O}$ vacancy formation energy reported in Table 2.

samples with equal proportion of the stoichiometric steps I and II, $a b$ initio calculations predict maximum $\mathrm{Pt}^{2+}$ coverage at the steps ( $\leq 33 \%$ of the step-edge sites) and maximum conversion of the $\mathrm{Pt}$ deposit to $\mathrm{Pt}^{2+}(\leq 33 \%$ of deposited $\mathrm{Pt})$ that are well below the experimental values $(50-80 \%$ of step-edge sites, up to $90 \%$ of deposited Pt, $c f$. Fig. 2a).

Most importantly, the calculations on the stoichiometric steps predict that Pt segregation, oxidation and the formation of the $\mathrm{Pt}^{2+}$ species are always accompanied by the reduction of surface Ce atoms from $\mathrm{Ce}^{4+}$ to $\mathrm{Ce}^{3+}$ (denoted in gray in Figs 3 and 4 ). The resulting concentration of the $\mathrm{Ce}^{3+}$ ions exceeds that of the $\mathrm{Pt}^{2+}$ ions by a factor of 2 . This is in stark contrast with the resonant PES measurements on our samples showing that the concentration of $\mathrm{Ce}^{3+}$ is considerably lower than the concentration of $\mathrm{Pt}^{2+}$ after annealing the samples (Fig. 2b). This indicates that $\mathrm{Pt}$ is preferentially oxidized by other mechanisms than the $\mathrm{Pt}^{0} / \mathrm{Ce}^{4+}$ redox couple.

\section{Table 2 | Minimum energy to remove an $\mathbf{O}$ atom from the $\mathrm{CeO}_{2}(111)$ surface obtained from DFT calculations.}

\begin{tabular}{lcc} 
O vacancy site & Pt step coverage & $\begin{array}{c}\text { O vacancy formation } \\
\text { energy (eV) }\end{array}$ \\
\hline Pt @ step II-S & $1 / 3$ & 3.8 \\
Pt @ step I-S & $1 / 3$ & 3.3 \\
$\mathrm{CeO}_{2}$ (111) & 0 & 2.5 \\
step II-S & $1 / 3$ & 2.0 \\
$\mathrm{Pt} @$ step I-O & $1 / 3$ & 1.9 \\
$\mathrm{Pt} @$ step II-O & 0 & 1.9 \\
step I-S & 1 & 1.8 \\
$\mathrm{Pt} @$ step I-O & 1 & 1.8 \\
Pt @ step II-O & 1.7 \\
\hline DFT, density functional theory. & \\
Results for steps I and II, and for Pt at steps I and II in the presence and absence of excess O. The \\
energies for Pt @ steps were calculated by removing the O atom marked by the * symbol in \\
Figs 3 and 4.
\end{tabular}

$\mathrm{Pt}^{2+}$ at $\mathrm{CeO}_{2}(111)$ step edges with excess $\mathrm{O}$. Agreement between the theory and the experiment can be achieved when taking into account the step edges in the presence of an excess of $\mathrm{O}$ atoms. Irrespective of the local step geometry and Pt coverage at the steps, we find that excess $\mathrm{O}$ atoms readily bind to $\mathrm{Pt}$ at the ceria steps and drive a rearrangement of the step morphology forming ionized $\mathrm{Pt}^{2+}$ species incorporated in the planar $\mathrm{PtO}_{4}$ moieties on both steps I and II (Fig. 3f,g). In the presence of excess of oxygen, Pt atoms bind stronger to the ceria step edges, with calculated binding energies up to $6.7 \mathrm{eV}$, which are higher than at the stoichiometric steps edges by $\sim 1.6 \mathrm{eV}$, and which are also higher than the cohesive energy of bulk metallic Pt (Table 1). This condition, which determines the stability of the $\mathrm{Pt}^{2+}$ species at steps with respect to metallic Pt clusters, is fulfilled only in the presence of excess oxygen at the steps. The computed electronic structure and density of states of the $\mathrm{PtO}_{4}$ moieties at the steps I-O and II-O (Supplementary Figs 5 and 6) confirm that the $\mathrm{Pt}^{0} \rightarrow \mathrm{Pt}^{2+}$ oxidation results from the ionic $\mathrm{Pt}-\mathrm{O}$ bond in the $\mathrm{PtO}_{4}$ planar units, and that $\mathrm{Ce}^{3+}$ ions do not form in agreement with the experimental evidence (Fig. 2b). The calculated maximum coverage of $\mathrm{Pt}^{2+}$ at the steps I-O and II-O is $100 \%$ (Fig. 4e,g and Supplementary Table 2), as interconnected assemblies of the $\mathrm{PtO}_{4}$ units can optimally fit the periodicities of both steps I and II at calculated Pt coverages at the step edges $1 / 3$, $2 / 3$ and 1 (Figs $3 \mathrm{f}, \mathrm{g}$ and $4 \mathrm{~d}-\mathrm{g}$ ). The presence of excess oxygen at steps therefore explains also the maximal $\mathrm{Pt}^{2+}$ ionization experimentally measured on the ceria-supported catalysts.

The stabilization of excess oxygen in the $\mathrm{PtO}_{4}$ moieties by the $\mathrm{Pt}^{2+}$ ions suggests an oxygen source for redox reactions and hence provides a link between the presence of highly dispersed ionic Pt species on ceria and the increased redox reactivity of $\mathrm{Pt}^{2+} / \mathrm{CeO}_{2}$ single-atom catalysts ${ }^{3}$. The oxygen buffering capacity of ceria-based catalysts is associated with easy oxygen vacancy formation. We calculate the vacancy formation energy on the clean $\mathrm{CeO}_{2}(111)$ terrace, on the stoichiometric steps and on the Pt-decorated steps (Table 2). Compared with the energy of $2.5 \mathrm{eV}$ calculated on the $\mathrm{CeO}_{2}(111)$ terrace, the energies required to remove an oxygen atom bound to $\mathrm{Pt}$ at the stoichiometric steps are $3.3 \mathrm{eV}$ (from the $\mathrm{PtO}_{4}$ unit at the step I-S) and $3.8 \mathrm{eV}$ (from the $\mathrm{PtO}_{4}$ unit at the step II-S; see Table 2). Pt segregation at the stoichiometric step edges yields the formation of strong Pt-O bonds and therefore hinders ceria O-buffering. Much lower energies are instead needed to remove the excess $\mathrm{O}$ incorporated in the $\mathrm{PtO}_{4}$ units at steps $\mathrm{I}-\mathrm{O}$ and II-O, where the $\mathrm{O}$ vacancy formation energy can be as low as $1.7 \mathrm{eV}$, lower or comparable to the values for the stoichiometric steps without Pt $(2.0-1.8 \mathrm{eV}$; 
Table 2$)^{30}$. This indicates that the dispersed $\mathrm{Pt}^{2+}$ ions can enhance the oxygen storage capacity of ceria-based catalysts by assisting the reversible storage of excess $\mathrm{O}$ atoms.

The single-ion nature of $\mathrm{Pt}^{2}+$ in the $\mathrm{PtO}_{4}$ units is preserved at all coverages, even when densely packed at the ceria step edges as interconnected $\mathrm{PtO}_{4}$ units. Indeed, the $\mathrm{Pt}$ charge state, its local electronic structure and the $\mathrm{O}$ vacancy formation energy of the densely packed $\mathrm{PtO}_{4}$ units are comparable to that of the isolated $\mathrm{PtO}_{4}$ units at the steps (Supplementary Fig. 7 and Table 2). The $\mathrm{PtO}_{4}$ units exhibit a large adaptability in stabilizing at different types of surface step edges, resulting in high effectiveness and capacity of the ceria surface to accommodate the $\mathrm{Pt}^{2+}$ ions. Regardless of the particular organization on the surface, the $\mathrm{PtO}_{4}$ units are also always accessible to the reactants. Thus, the square-planar $\mathrm{PtO}_{4}$ units carrying monodispersed $\mathrm{Pt}^{2}+$ ions can be considered elementary building blocks of single-atom Pt-ceria catalysts.

\section{Discussion}

On large-area samples, $\mathrm{Pt}^{2+}$ ions on ceria show exceptional reactivity with minimum $\mathrm{Pt}$ load in important applications: water-gas shift reaction ${ }^{3}$, hydrogen oxidation on the anode of proton-exchange membrane fuel cell ${ }^{11}$ and in the three-way catalyst converter ${ }^{10}$. In these applications, $\mathrm{Pt}^{2+}$ ions on ceria exhibit long-term stability under realistic reaction conditions of elevated temperatures and ambient pressure of reactant gases $3,31,32 . \mathrm{Pt}^{2+}$ in large-area samples is routinely identified with PES. Complementary measurements with extended X-ray absorption fine structure (EXAFS) ${ }^{33}$ and high-resolution transmission electron microscopy ${ }^{3,4,31,32}$ confirm the absence of three-dimensional $\mathrm{Pt}$ or $\mathrm{PtO}_{x}$ clusters and, in agreement with the advanced PES measurements ${ }^{34}$, identify $\mathrm{Pt}^{2+}$ as highly dispersed surface species on ceria ${ }^{32}$.

Our present study identifies the stabilization of monodispersed $\mathrm{Pt}^{2+}$ ions with one particular defect site on the ceria surface-the monoatomic step edge-and excludes the stabilization of $\mathrm{Pt}^{2+}$ on the oxygen vacancies. Monodispersed $\mathrm{Pt}$ on ceria is observed to be effective in incorporating excess oxygen even in the unfavourable conditions of UHV experiment. Excess oxygen and $\mathrm{Pt}^{2}+$ arrange in the square-planar $\mathrm{PtO}_{4}$ moieties decorating different types of the surface steps at coverages up to one $\mathrm{PtO}_{4}$ per one step-edge $\mathrm{Ce}$ atom. The excess oxygen can be easily detached, indicating enhancement of the redox properties of ceria loaded with the $\mathrm{Pt}^{2+}$ ions. Adjusting the step density and the $\mathrm{Pt}$ load on the model $\mathrm{CeO}_{2}(111)$ surface allows maximizing the coverage of $\mathrm{Pt}^{2+}$, while suppressing the nucleation of metallic $\mathrm{Pt}^{\mathrm{O}}$ clusters. In the present experiment, we achieve surface coverage of $\mathrm{Pt}^{2+} 0.05 \mathrm{ML}$. A further increase of the completely monodispersed ionized $\mathrm{Pt}^{2}+$ coverage to $0.1 \mathrm{ML}$ can be expected.

Step edges on ceria have been previously identified as preferred nucleation sites for supported metal clusters ${ }^{20,21,27,28,35-37}$. Our present study highlights the property of the step edges on ceria to provide specific structural and electronic environments for selective formation of monodispersed, thermally and chemically stable $\mathrm{Pt}^{2+}$ ions. The step edges represent intrinsic defects ubiquitously present on nanostructured ceria surfaces ${ }^{4,38}$; our results are thus applicable for the interpretation of the properties and the optimization of the $\mathrm{Pt}^{2+}$ load on large-area ceria supports $3,10,11$. More generally, the step edges may represent a common type of adsorption sites providing stabilization for monodispersed metal atoms and ions in any oxide-supported single-atom catalysts ${ }^{15,39}$. Our results therefore introduce important concepts of step reactivity ${ }^{40}$ and step engineering ${ }^{13,14}$ in understanding the stability, the activity and in designing new single-atom catalysts.

\section{Methods}

Experiment. The experiments were performed on surface science apparatuses in Surface Science Laboratory in Prague (STM, laboratory X-ray PES (XPS) with $h v=1,487 \mathrm{eV}(\mathrm{Al} \mathrm{K} \alpha)$, low-energy electron diffraction) and at the Materials Science Beamline in Trieste (PES with $h v=22-1,000 \mathrm{eV}$ (synchrotron), laboratory XPS with $h v=1,487 \mathrm{eV}(\mathrm{Al} \mathrm{K} \alpha)$ and low-energy electron diffraction).

Preparation of the ceria substrates. The ceria layers and their Pt loading were prepared using the same procedures and parameters in both laboratories, and investigated by surface science methods in situ without exposing to air. The procedures and parameters of all samples are summarized in Supplementary Table 1. The ceria layers were prepared by deposition of Ce metal (Ce wire $99.9 \%$, Goodfellow Cambridge Ltd) from Ta or Mo crucible heated by electron bombardment on clean $\mathrm{Cu}(111)$ substrate $(\mathrm{MaTecK} \mathrm{GmbH})$ in a background pressure of $5 \times 10^{-5} \mathrm{~Pa}$ of $\mathrm{O}_{2}(5.0$, Linde $\mathrm{AG})$. The growth rate of $\mathrm{CeO}_{2}$ was $6 \mathrm{ML}$ per hour. Varying densities of $1 \mathrm{ML}$ high steps on the prepared $\mathrm{CeO}_{2}$ layers were obtained by growth of $\mathrm{CeO}_{2}$ at constant substrate temperature 423 or $523 \mathrm{~K}$ (Method I in Supplementary Table 1) or linearly increasing substrate temperature from room temperature to $723 \mathrm{~K}$ (Method II in Supplementary Table 1) ${ }^{16}$. For experiments in Fig. 1, the ordered, fully oxidized layer of $\mathrm{CeO}_{2}$ (Fig. 1a,b) and the ordered reduced layer of $\mathrm{CeO}_{1.7}$ (Fig. 1d,e) were obtained by approach published in ref. 17 that yields the lowest step density. In this approach, first, fully reduced $\mathrm{Ce}_{2} \mathrm{O}_{3}$ layer is prepared by depositing metallic $\mathrm{Ce}$ on a $\mathrm{CeO}_{2}$ layer and annealing in vacuum. Subsequently, the $\mathrm{Ce}_{2} \mathrm{O}_{3}$ layer is exposed to a controlled dose of $\mathrm{O}_{2}$ at $5 \times 10^{-5} \mathrm{~Pa}$ and annealed to obtain desired stoichiometry $\mathrm{CeO}_{1.7}$ or $\mathrm{CeO}_{2}$ (Method III in Supplementary Table 1). The $\mathrm{CeO}_{2}$ surface imaged in Fig. 1g,h was obtained by depositing $0.3 \mathrm{ML} \mathrm{CeO}$ on the $\mathrm{CeO}_{2}(111)$ substrate as in Fig. 1a, forming small ML-high islands. This homoepitaxy of $\mathrm{CeO}_{2}$ on $\mathrm{CeO}_{2}$ yields clearly arranged samples with high step density (Method IV in Supplementary Table 1).

Characterization of the ceria substrates. The thickness of the ceria layers was determined from the attenuation of the substrate $\mathrm{Cu} 2 p_{3 / 2}$ XPS signal measured at $h v=1,487 \mathrm{eV}$. For calculations, we used inelastic mean free path of electrons in $\mathrm{CeO}_{2} 11.2 \AA$. The thickness of the ceria layers was set between 20 and $40 \AA$ or 7 and $12 \mathrm{ML}$ with $1 \mathrm{ML}$ corresponding to $3.1 \AA$, the distance between $\mathrm{Ce}(111)$ atomic planes of $\mathrm{CeO}_{2}$. In this range of thickness, the coverage of the $\mathrm{Cu}$ substrate by ceria ranges between 97 and $100 \%$ (ref. 17). For determining the density of 1 ML high steps, we use a semi-automated procedure when the first step outlines are marked in STM images manually. Step outlines are then mapped onto a properly scaled and rotated hexagonal mesh of surface $\mathrm{Ce}$ atoms. The atoms that are closest to the outlines are automatically identified as step-edge atoms and their density evaluated in ML. The error in determining the density of $1 \mathrm{ML}$ high steps is estimated to be $\pm 10 \%$ and is marked in Fig. 2a.

Preparation of the Pt deposit. Pt was deposited on the ceria layers from a Pt wire (99.99\%, MaTecK GmbH) heated by electron bombardment. Pt was deposited on the sample surface at $300 \mathrm{~K}$ and subsequently stabilized by increasing the sample temperature to $700 \mathrm{~K}$ at the rate $2 \mathrm{Ks} \mathrm{s}^{-1}$. Both Pt deposition and annealing proceeded in the UHV background pressure $5 \times 10^{-8} \mathrm{~Pa}$ or below. The thermal treatment supports the ionization of $\mathrm{Pt}$ to $\mathrm{Pt}^{2+}$.

Characterization of the Pt deposit. The amount of Pt was calculated from the deposition time after calibrating the constant evaporation rate of the Pt evaporator. The evaporation rate was determined by a Quartz Crystal Microbalance and/or in a dedicated experiment from the thickness of 4-ML-thick Pt layers on $\mathrm{CeO}_{2}(111)$ / $\mathrm{Cu}(111)$ determined by attenuation of the substrate $\mathrm{Cu} 2 p_{3 / 2}$ XPS signal measured at $h v=1,487 \mathrm{eV}$. This dedicated experiment was used to correlate Pt evaporation rates between the two experimental apparatuses. For calculations, we used inelastic mean free path of electrons in Pt $8 \AA$. The fraction of $\mathrm{Pt}^{2+}$ after thermal treatment was determined by fitting the ionic $\mathrm{Pt}^{2+}$ and neutral $\mathrm{Pt}^{0}$ component in the PES Pt $4 f$ spectrum measured at $h v=180 \mathrm{eV}$ ( $c f$. Fig. $1 c, \mathrm{f}, \mathrm{i})$. The error in determining the $\mathrm{Pt}$ and $\mathrm{Pt}^{2+}$ amounts on the studied samples is $\pm 20 \%$ and is marked in Fig. 2a. This error represents the calibration error of the Pt evaporation rate.

Resonant PES. Reduction of the ceria surface after deposition of Pt and thermal treatment was determined with resonant PES of Ce $4 f$ state. We determine the so-called resonant enhancement ratio (RER) as defined in refs 41,42 from measurements of intensities of $\mathrm{Ce}^{3+}$ and $\mathrm{Ce}^{4+}$ components of valence-band resonant PES Ce $4 f$ spectra of $\mathrm{CeO}_{2}$ measured off-resonance $(h v=115 \mathrm{eV})$ and onresonance $\left(h v=121.4 \mathrm{eV}\right.$ for the $\mathrm{Ce}^{3+}$ component and $h v=124.8 \mathrm{eV}$ for the $\mathrm{Ce}^{4+}$ component). The value of resonant enhancement ratio represents an upper estimate of the concentration of $\mathrm{Ce}^{3+}$ ions on the ceria surface and is plotted in Fig. $2 b^{17,42}$.

STM imaging. STM measurements were performed with commercial Pt-Ir tips (Unisoku). STM imaging of $\mathrm{CeO}_{2}(111)$ and $\mathrm{Pt} / \mathrm{CeO}_{2}(111)$ films was available only 
via unoccupied states. We used sample voltages $2.5-3.5 \mathrm{~V}$ and tunnelling currents 25-75 pA.

Theory. All calculations were based on the DFT and were performed using the spin-polarized GGA $+U$ approach $^{43}$, employing the Perdew-Burke-Ernzerhof exchange-correlation functional ${ }^{44}$ and ultrasoft pseudopotentials ${ }^{45}$. The spinpolarized Kohn-Sham equations were solved with a plane-wave basis set and the Fourier representation of the charge density was limited by kinetic cutoffs of 40 and $320 \mathrm{Ry}$, respectively. The Quantum-ESPRESSO computer package was used in all the calculations ${ }^{46}$. In the Hubbard $U$ term, the occupations of the $f$-orbitals were defined in terms of atomic wave function projectors and the value of the parameter $U$ was set to $4.5 \mathrm{eV}$, following our previous studies ${ }^{47,48}$.

Slab models. The ceria $(111)$ surfaces were modelled with periodic $(3 \times 3)$ slabs being three $\mathrm{CeO}_{2}$ ML thick and separated by more than $10 \AA$ of vacuum in the direction perpendicular to the surface. The Brillouin zone was sampled at Gamma point. In the present work, we considered two low-energy ML-high steps that we label following ref. 25 as step I and step II. The edge of both step I and step II steps are oriented along the $[1 \overline{1} 0]$ direction. These surface steps were modelled with vicinal surfaces described with monoclinic periodic slabs separated by $>10 \AA$ of vacuum in the direction perpendicular to the (111) terrace. The dimensions of the cells were $17.97 \times 11.67 \AA^{2}$ along the $[1 \overline{1} 2]$ and $[1 \overline{1} 0]$ directions (step I) and $15.72 \times 11.67 \AA^{2}$ along the $[1 \overline{1} 2]$ and $[1 \overline{1} 0]$ (step II). All the vicinal surfaces slabs included three $\mathrm{CeO}_{2} \mathrm{ML}$. This thickness was shown to be sufficient to calculate the structural and thermodynamic properties of these steps ${ }^{25}$. The complete set of surface structures and systems considered in this work is listed in the Supplementary Note 1 . All these systems were structurally optimized according to the Hellmann-Feynman forces. During the geometry optimization, the atomic positions of the lowermost $\mathrm{CeO}_{2} \mathrm{ML}$ were constrained, as well as those of the $\mathrm{Ce}$ atoms in the central ML, except for the Ce atoms below the step edge.

Energetics. Binding energies were computed as $1 / N_{\mathrm{Pt}}\left(E_{\mathrm{slab}}+N_{\mathrm{Pt}} E_{\mathrm{Pt}}-E_{\mathrm{slab} / \mathrm{Pt}}\right)$, where $E_{\text {slab/Pt }}$ is the total energy of the ceria slab containing $N_{\mathrm{Pt}}$ atoms of $\mathrm{Pt}, E_{\text {slab }}$ is the total energy of the corresponding relevant (stoichiometric, reduced or oxidized) Pt-free ceria slab and $E_{\mathrm{Pt}}$ is the total energy of a $\mathrm{Pt}$ atom in vacuum. The energies required to form an oxygen vacancy $\mathrm{Ov}$ were calculated as $\left(E_{\mathrm{slab} / \mathrm{Ov}}+1 / 2 E_{\mathrm{O} 2}-E_{\mathrm{slab}}\right)$, where $E_{\mathrm{slab}}$ $\mathrm{O}_{\mathrm{v}}$ and $E_{\text {slab }}$ are the total energies of the ceria supercell with and without the $\mathrm{O}$ vacancy, respectively, whereas $E_{\mathrm{O} 2}$ is the total energy of a gas-phase $\mathrm{O}_{2}$ molecule compensated for the known overbinding predicted by (semi)local functionals for $\mathrm{O}_{2}$.

\section{References}

1. Yang, X. et al. Single-atom catalysts: a new frontier in heterogeneous catalysis. Acc. Chem. Res. 46, 1740-1748 (2013).

2. Flytzani-Stephanopoulos, M. \& Gates, B. C. Atomically dispersed supported metal catalysts. Annu. Rev. Chem. Biomol. Eng. 3, 545-574 (2012).

3. Fu, Q., Saltsburg, H. \& Flytzani-Stephanopoulos, M. Active nonmetallic Au and Pt species on ceria-based water-gas shift catalysts. Science 301, 935-938 (2003).

4. Bruix, A. et al. Maximum noble-metal efficiency in catalytic materials: atomically dispersed surface platinum. Angew. Chem. Int. Ed. 53, 10525-10530 (2014).

5. Qiao, B. et al. Single-atom catalysis of $\mathrm{CO}$ oxidation using $\mathrm{Pt}_{1} / \mathrm{FeO}_{\mathrm{x}}$. Nat. Chem. 3, 634-641 (2011).

6. Novotný, Z. et al. Ordered array of single adatoms with remarkable thermal stability: $\mathrm{Au} / \mathrm{Fe}_{3} \mathrm{O}_{4}(001)$. Phys. Rev. Lett. 108, 216103 (2012).

7. Parkinson, G. S. et al. Carbon monoxide-induced adatom sintering in a Pd- $\mathrm{Fe}_{3} \mathrm{O}_{4}$ model catalyst. Nat. Mater. 12, 724-728 (2013).

8. Bliem, R. et al. Subsurface cation vacancy stabilization of the magnetite (001) surface. Science 346, 1215-1218 (2014).

9. Li, F., Li, Y., Zeng, X. C. \& Chen, Z. Exploration of high-performance single-atom catalysts on support $\mathrm{M}_{1} / \mathrm{FeO}_{x}$ for $\mathrm{CO}$ oxidation via computational study. ACS Catal. 5, 544-552 (2015).

10. Hatanaka, M. et al. Ideal Pt loading for a $\mathrm{Pt} / \mathrm{CeO}_{2}$-based catalyst stabilized by a Pt-O-Ce bond. Appl. Catal. B Environ. 99, 336-342 (2010).

11. Fiala, R. et al. Proton exchange membrane fuel cell made of magnetron sputtered $\mathrm{Pt}-\mathrm{CeO}_{\mathrm{x}}$ and $\mathrm{Pt}-\mathrm{Co}$ thin film catalysts. J. Power Sources 273, 105-109 (2015).

12. Gong, X.-Q., Selloni, A., Batzill, M. \& Diebold, U. Steps on anatase $\mathrm{TiO}_{2}(101)$. Nat. Mater. 5, 665-670 (2006).

13. Barth, J. V, Costantini, G. \& Kern, K. Engineering atomic and molecular nanostructures at surfaces. Nature 437, 671-679 (2005).

14. Vang, R. T. et al. Controlling the catalytic bond-breaking selectivity of $\mathrm{Ni}$ surfaces by step blocking. Nat. Mater. 4, 160-162 (2005).

15. Gong, X., Selloni, A., Dulub, O., Jacobson, P. \& Diebold, U. Small Au and Pt clusters at the anatase $\mathrm{TiO}_{2}(101)$ surface: behavior at terraces, steps, and surface oxygen vacancies. J. Am. Chem. Soc. 130, 370-381 (2008).

16. Dvořák, F. et al. Adjusting morphology and surface reduction of $\mathrm{CeO}_{2}(111)$ thin films on $\mathrm{Cu}(111)$. J. Phys. Chem. C 115, 7496-7503 (2011).
17. Duchon, T. et al. Ordered phases of reduced ceria as epitaxial films on $\mathrm{Cu}(111)$. J. Phys. Chem. C 118, 357-365 (2014).

18. Zhou, Y., Perket, J. M. \& Zhou, J. Growth of Pt nanoparticles on reducible $\mathrm{CeO}_{2}$ (111) thin films: effect of nanostructures and redox properties of ceria. J. Phys. Chem. C 114, 11853-11860 (2010).

19. Shao, X., Jerratsch, J.-F., Nilius, N. \& Freund, H.-J. Probing the $4 \mathrm{f}$ states of ceria by tunneling spectroscopy. Phys. Chem. Chem. Phys. 13, 12646-12651 (2011).

20. Lu, J.-L., Gao, H.-J., Shaikhutdinov, S. \& Freund, H.-J. Morphology and defect structure of the $\mathrm{CeO}_{2}(111)$ films grown on $\mathrm{Ru}(0001)$ as studied by scanning tunneling microscopy. Surf. Sci. 600, 5004-5010 (2006).

21. Zhou, Y. \& Zhou, J. Interactions of Ni nanoparticles with reducible $\mathrm{CeO}_{2}(111)$ thin films. J. Phys. Chem. C 116, 9544-9549 (2012).

22. Mullins, D. R. et al. Water dissociation on $\mathrm{CeO}_{2}(100)$ and $\mathrm{CeO}_{2}(111)$ thin films. J. Phys. Chem. C 116, 19419-19428 (2012).

23. Bruix, A. et al. A new type of strong metal-support interaction and the production of $\mathrm{H}_{2}$ through the transformation of water on $\mathrm{Pt} / \mathrm{CeO}_{2}(111)$ and $\mathrm{Pt} / \mathrm{CeO}_{\mathrm{x}} / \mathrm{TiO}_{2}(110)$ catalysts. J. Am. Chem. Soc. 134, 8968-8974 (2012).

24. Negreiros, F. R. \& Fabris, S. Role of cluster morphology in the dynamics and reactivity of subnanometer Pt clusters supported on ceria surfaces. J. Phys. Chem. C 118, 21014-21020 (2014).

25. Kozlov, S. M., Viñes, F., Nilius, N., Shaikhutdinov, S. \& Neyman, K. M. Absolute surface step energies: accurate theoretical methods applied to ceria nanoislands. J. Phys. Chem. Lett. 3, 1956-1961 (2012).

26. Torbrügge, S., Cranney, M. \& Reichling, M. Morphology of step structures on $\mathrm{CeO}_{2}(111)$. Appl. Phys. Lett. 93, 073112 (2008).

27. James, T. E., Hemmingson, S. L. \& Campbell, C. T. Energy of supported metal catalysts: from single atoms to large metal nanoparticles. ACS Catal. 5, 5673-5678 (2015).

28. James, T. E., Hemmingson, S. L., Ito, T. \& Campbell, C. T. Energetics of $\mathrm{Cu}$ adsorption and adhesion onto reduced $\mathrm{CeO}_{2}(111)$ surfaces by calorimetry. J. Phys. Chem. C 119, 17209-17217 (2015).

29. Colussi, S. et al. Nanofaceted Pd-O sites in Pd-Ce surface superstructures: Enhanced activity in catalytic combustion of methane. Angew. Chem. Int. Ed. 48, 8481-8484 (2009).

30. Kozlov, S. M. \& Neyman, K. M. O vacancies on steps on the $\mathrm{CeO}_{2}(111)$ surface Phys. Chem. Chem. Phys. 16, 7823-7829 (2014).

31. Fiala, R. et al. Pt-CeO $\mathrm{x}_{\mathrm{x}}$ thin film catalysts for PEMFC. Catal. Today 240, 236-241 (2015)

32. Hatanaka, M. et al. Reversible changes in the Pt oxidation state and nanostructure on a ceria-based supported Pt. J. Catal. 266, 182-190 (2009).

33. Nagai, Y. et al. Sintering inhibition mechanism of platinum supported on ceria-based oxide and Pt-oxide-support interaction. J. Catal. 242, 103-109 (2006).

34. Matolín, V. et al. Platinum-doped $\mathrm{CeO}_{2}$ thin film catalysts prepared by magnetron sputtering. Langmuir 26, 12824-12831 (2010).

35. Zhou, J., Baddorf, A. P., Mullins, D. R. \& Overbury, S. H. Growth and characterization of $\mathrm{Rh}$ and $\mathrm{Pd}$ nanoparticles on oxidized and reduced $\mathrm{CeO}_{\mathrm{x}}(111)$ thin films by scanning tunneling microscopy. J. Phys. Chem. C 112, 9336-9345 (2008).

36. Zhou, Y., Perket, J. M. \& Zhou, J. Growth of Pt nanoparticles on reducible $\mathrm{CeO}_{2}(111)$ thin films: effect of nanostructures and redox properties of ceria J. Phys. Chem. C 114, 11853-11860 (2010).

37. Zhou, Y. \& Zhou, J. Growth and sintering of $\mathrm{Au}-\mathrm{Pt}$ nanoparticles on oxidized and reduced $\mathrm{CeO}_{\mathrm{x}}(111)$ thin films by scanning tunneling microscopy. J. Phys. Chem. Lett. 1, 609-615 (2010).

38. Sayle, T. X. T., Parker, S. C. \& Sayle, D. C. Oxidising $\mathrm{CO}$ to $\mathrm{CO}_{2}$ using ceria nanoparticles. Phys. Chem. Chem. Phys. 7, 2936-2941 (2005).

39. Castellani, N. J., Branda, M. M., Neyman, K. M. \& Illas, F. Density functional theory study of the adsorption of Au atom on cerium oxide: effect of lowcoordinated surface sites. J. Phys. Chem. C 113, 4948-4954 (2009).

40. Zambelli, T., Wintterlin, J., Trost, J. \& Ertl, G. Identification of the 'active sites' of a surface-catalyzed reaction. Science 273, 1688-1690 (1996).

41. Matolín, V. et al. Water interaction with $\mathrm{CeO}_{2}(111) / \mathrm{Cu}(111)$ model catalyst surface. Catal. Today 181, 124-132 (2012).

42. Mullins, D. R. The surface chemistry of cerium oxide. Surf. Sci. Rep. 70, 42-85 (2015).

43. Cococcioni, M. \& de Gironcoli, S. Linear response approach to the calculation of the effective interaction parameters in the LDA + U method. Phys. Rev. B 71, 035105 (2005).

44. Perdew, J. P. J., Burke, K. \& Ernzerhof, M. Generalized gradient approximation made simple. Phys. Rev. Lett. 77, 3865-3868 (1996).

45. Vanderbilt, D. Soft self-consistent pseudopotentials in a generalized eigenvalue formalism. Phys. Rev. B 41, $7892-7895$ (1990).

46. Giannozzi, P. et al. QUANTUM ESPRESSO: a modular and open-source software project for quantum simulations of materials. J. Phys. Condens. Matter 21, 395502 (2009). 
47. Fabris, S., de Gironcoli, S., Baroni, S., Vicario, G. \& Balducci, G. Taming multiple valency with density functionals: a case study of defective ceria. Phys. Rev. B 71, 041102 (2005).

48. Fabris, S., Vicario, G., Balducci, G., De Gironcoli, S. \& Baroni, S. Electronic and atomistic structures of clean and reduced ceria surfaces. J. Phys. Chem. B 109, 22860-22867 (2005).

\section{Acknowledgements}

This work was supported by Czech Science Foundation (contract numbers 15-06759S and 13-10396S), and by the European Union via the FP7-NMP-2012 project chipCAT under contract number 310191 and the EU FP7 COST action CM1104. A.T. acknowledges the support of the Grant Agency of the Charles University, contract number 2048514. S.F. acknowledges the support provided by the Humboldt Foundation through a Friedrich Wilhelm Bessel Research Award. The high-performance computing resources were gratefully provided by ISCRA initiative of CINECA. CERIC-ERIC consortium is acknowledged for financial support.

\section{Author contributions}

F.D., A.T., M.V., T.S., I.M., J.M. and V.M. designed and performed the experiments. M.F.C., N.-D.T., F.R.N. and S.F. performed the DFT calculations. All authors interpreted the experimental and computational results. J.M., S.F., T.S., I.M. and V.M. wrote the manuscript. F.D., A.T., J.M., I.M., V.M. and S.F. provided funding.

\section{Additional information}

Supplementary Information accompanies this paper at http://www.nature.com/ naturecommunications

Competing financial interests: The authors declare no competing financial interests.

Reprints and permission information is available online at http://npg.nature.com/ reprintsandpermissions/

How to cite this article: Dvořák, F. et al. Creating single-atom Pt-ceria catalysts by surface step decoration. Nat. Commun. 7:10801 doi: 10.1038/ncomms10801 (2016).

This work is licensed under a Creative Commons Attribution 4.0 International License. The images or other third party material in this article are included in the article's Creative Commons license, unless indicated otherwise in the credit line; if the material is not included under the Creative Commons license, users will need to obtain permission from the license holder to reproduce the material. To view a copy of this license, visit http://creativecommons.org/licenses/by/4.0/ 